\title{
Preguntas en los libros de texto de Ciencias Naturales de Educación Primaria: ¿Procesando o reproduciendo contenidos?
}

\author{
José Manuel Pérez-Martín. Universidad Autónoma de Madrid \\ $M^{a}$ Tentudía Calurano-Tena. Universidad Autónoma de Madrid \\ Cristina Martín-Aguilar. Universidad Autónoma de Madrid \\ Tamara Esquivel-Martín. Universidad Autónoma de Madrid \\ Beatriz Bravo-Torija. Universidad Autónoma de Madrid
}

Recepción: 03.03.2019 | Aceptado: 15.10.2019

Correspondencia a través de ORCID: José Manuel Pérez Martín

Citar: Pérez-Martín, JM., Calurano-Tena, MT., Martín-Aguilar, C., Esquivel-Martín, T. y Bravo-Torija, B. (2019). Preguntas en los libros de texto de Ciencias Naturales de Educación Primaria: ¿Procesando o reproduciendo contenidos? ReiDoCrea, 8, 186- 201.

Agradecimientos. Esta investigación y la difusión de sus resultados está financiada por los proyectos de 1+D+i EDU2017-82688-P (JMPM y TEM) y EDU2015-66643-C2-2-P (BBT), y se encuadra en la investigación que desarrollan BBT, JMPM y TEM en el marco de la Cátedra UNESCO en Educación para la Justicia Social en la UAM.

\begin{abstract}
Resumen: Los libros de texto son el recurso didáctico de primera elección en Educación Primaria, aunque su papel está siendo cuestionado. Nuestro estudio valora el rol de este recurso como herramienta de evaluación, analizando los tipos de preguntas que aparecen en el bloque de los ecosistemas de siete libros de texto publicados en el marco legislativo de las tres últimas leyes educativas españolas (LOGSE, LOE y LOMCE). Las preguntas presentes en los libros se han clasificado en fácticas (memorísticas) y de proceso (productivas). Los resultados demuestran que la evaluación en España se ha basado tradicionalmente en preguntas fácticas que promueven aprendizajes memorísticos, pero el número de preguntas de proceso en los libros de texto ha aumentado tras la publicación de la LOMCE. Sin embargo, aún queda un largo camino por recorrer hasta que los libros de texto propongan verdaderas preguntas para pensar.
\end{abstract}

Palabras clave: Enseñanza Primaria | Enseñanza de Biología

Natural Science questions in Primary Education textbooks: Processing or reproducing contents?

Abstract: Textbooks are the didactic resource of choice in Primary Education, although their role is being questioned. Our study assesses the role of this resource as an evaluation tool, analyzing the types of questions that appear in the block of ecosystems of seven textbooks published in the legislative framework of the last three Spanish educational laws (LOGSE, LOE and LOMCE). The questions of that books have been classified as factual questions (memory) and process questions (productive). Results show that evaluation in Spain has traditionally been based on the first ones that promote rote learning, but the number of process questions in the textbooks has increased after the LOMCE's publication. However, there is still a long way to go towards reaching the appearance of true thought-provoking questions in textbooks.

Keywords: Elementary Education | Biology Education

\section{Introducción}

Los cambios legislativos en España desde los primeros años del siglo XXI han promovido una formación más enfocada a desarrollar competencias, sin descuidar los contenidos conceptuales (García González y Pérez Martín, 2016; MECD, 2013). La Ley Orgánica General del Sistema Educativo (LOGSE) entró en vigor en el curso 1991-1992. En ella se proponía una ordenación del sistema educativo que lo modernizaba respecto de la ley anterior, que venía del período predemocrático y había sufrido varias modificaciones. Con el paso del tiempo, las necesidades educativas en 2006 condujeron a promulgar la Ley Orgánica de Educación (LOE), que introdujo por primera vez ideas sobre la educación y la formación de los estudiantes en el marco de las competencias, incluida la competencia científica. En la actualidad, la Ley Orgánica para la Mejora de la Calidad Educativa (LOMCE) ha modificado la organización de diferentes aspectos, pero continúa con la filosofía de la adquisición de conocimientos, competencias y 
capacidades durante la formación obligatoria (MECD, 2013). Concretamente en el caso de la competencia matemática y competencias básicas en ciencia y tecnología, se persigue que ayuden a aplicar los contenidos de las Ciencias de forma integral, permitiendo la resolución de problemas de forma eficaz en Educación Primaria (EP), como se indica en el Artículo 7.1 de la LOMCE (MECD, 2013). En este sentido, estas ideas inspiradas por la ley en vigor parecen sugerir que el ejercicio docente debe reducir las prácticas memorísticas y promover aprendizajes más prácticos y capacitantes para la vida cotidiana.

Esta propuesta legislativa está en la línea de estudios internacionales que nos instan a promover cambios en la forma de enseñar Ciencias (Rocard et al., 2007), ya que existe una gran desafección por su aprendizaje (Robles, Solbes, Cantó y Lozano, 2015; Solbes, Montserrat y Furió, 2007), que se ha relacionado con diferentes problemas didácticos. Entre ellos, cabe destacar la falta de vinculación entre lo que se enseña y la vida cotidiana (Solbes, 2011), así como la aplicación de metodologías tradicionales y memorísticas en su enseñanza. Desde la universidad, se promueve el uso de metodologías activas y se insta a los docentes en formación y en ejercicio a que las pongan en práctica en sus aulas. Sin embargo, esta insistencia ni siquiera se ajusta a la práctica que ejecutamos en la universidad, como muestran los resultados obtenidos por García-Carmona, Cruz-Guzmán y Criado (2014).

Tanto es así que, aunque actualmente los docentes disponen de numerosos materiales con los que preparar sus clases en cualquier nivel educativo, el libro de texto sigue siendo el recurso de primera elección en las aulas (Braga Blanco y Berver Domínguez, 2016; Cantó, Pro y Solbes, 2016; Carmen y Jiménez Aleixandre, 2010; López-Valentín y Guerra-Ramos, 2013). Uno de los motivos para la preponderancia del uso del libro de texto que argumentan diferentes autores han sido las cuestiones socioeconómicas y políticas (Beas, 1999; López Valentín y Guerra Ramos, 2013; Torres Santomé, 1989). Sin embargo, existen otros motivos que se sustentan en decisiones pedagógicas. En primer lugar, hay que destacar que, según la Asociación Nacional de Editores de Libros y material de Enseñanza (2013), el $71,9 \%$ de los padres consideran indispensable el uso de libro de texto en la formación de sus hijos. Además, el $81,3 \%$ de docentes reconocen utilizarlo bastante o mucho (ANELE, 2013), creando un documento de uso diario que permite aplicar la legislación de forma concreta y sencilla (Blanco Braga y Belver Domínguez, 2016; García, 2001; López Valentín y Guerra Ramos, 2013; Quiroz, 2001), por lo que se convierte en el currículum real (Altbach y Kelly, 1988). Además, estos materiales realizan una criba de lo que deben saber los estudiantes, sin necesidad de que el docente lo tenga que decidir (Puelles, 2000; Torres, 1994). Aunque no todos los profesores utilizan los libros de texto de la misma forma (Carmen, 2010; Carmen y Jiménez Aleixandre, 2010), diferentes investigaciones muestran que el libro de texto pauta la acción docente en muchos casos (Area, 2009; López Valentín y Guerra Ramos, 2013). Esto provoca que los docentes se vuelvan acríticos con los contenidos y la metodología, y desprofesionaliza al profesorado (Apple, 1993; Martínez Bonafé, 2002), ya que son otros profesionales los que definen los quehaceres diarios en la formación de los estudiantes. Esta situación no favorece el desarrollo profesional del docente (Braga Blanco y Belver Domínguez, 2016), ya que permite que profesores con carencias formativas se escuden en este recurso didáctico o incluso que no perciban la necesidad de la formación continua (Arriassecq y Greca, 2004; Megid Neto y Fracalanza, 2003; Monereo Font, 2010).

Todo ello justifica que los docentes promuevan su utilización, provocando que nadie dude de su contenido y legitimándolo como la fuente rigurosa de conocimiento (Certad Villaroel, 2011; Occelli y Valeiras, 2013). En cambio, el rigor de los contenidos y su didáctica está cada vez más en entredicho (Blanco Braga y Belver Domínguez, 2016; Calvo Pascual y Martín Sánchez, 2005; García González y Pérez Martín, 2016; López 
Valentín y Guerra Ramos, 2013; Martínez Losada y García Barros, 2003). Tanto es así que la revisión realizada por Occelli y Valeiras (2013) sugiere que se debería analizar cómo se trabaja en las aulas con los libros de texto, ya que presentan generalmente una pauta muy cerrada en los procedimientos científicos que deberían desarrollar para la correcta alfabetización científica (resolución de problemas, experimentación, indagación, trabajo en grupo, búsqueda de información y comunicación de los resultados, etc.), así como desatiende la atención a diversidad. Además, reseñamos el análisis relativo a las actividades de evaluación, concretamente al tipo de preguntas. Diferentes autores (Concari y Giorgi, 2002; Costa Amaral, Da Silva y Maciel, 2009; Ferreiro y Occelli, 2008; García González y Pérez Martín, 2016; García-Rodeja Gayoso, 1997; Patiño Garzón, Vera Márquez y Meisel Donoso, 2010; Quílez, 2006) han puesto de manifiesto que la mayoría de las actividades de los libros de texto no fomentan la interpretación de lo memorizado, ya que frecuentemente requieren al alumno que copie literalmente los contenidos previamente presentados. Este tipo de preguntas las podemos definir como fácticas ya que para su respuesta sólo se requiere memorizar y reproducir el contenido y usan la ciencia como producto (Furman, Poenitz y Podestá, 2012). Estas autoras definen, en contraposición, un segundo tipo de preguntas, las que requieren de un procesamiento de lo aprendido, en las cuales se usa la ciencia como proceso, fomentando la reflexión y resolución de problemas. Este tipo de preguntas han sido denominadas por otros autores como "preguntas mediadoras" (Márquez, Bonil y Pujol, 2005), "preguntas que ayudan a aprender" (Márquez y Roca, 2006) o "preguntas para pensar" (Andersson y Gullberg, 2014), en inglés thought-provoking questions, todas ellas muy útiles para desarrollar el pensamiento crítico.

El análisis de libros de texto en Didáctica de las Ciencias se ha centrado en múltiples aspectos como los contenidos, las competencias, las imágenes e incluso en las actividades (Borges Fernandes, Pires y Delgado-Iglesias, 2017; López-Manjón y Postigo, 2016; Martínez Losada y García Barros, 2003; Occelli y Valeiras, 2013; Rodríguez Moreno, Pro Bueno y Molina Jaén, 2018; Urones, Escobar y Vacas, 2017). Sin embargo, en pocas ocasiones se realizan clasificaciones sencillas de las preguntas de evaluación que formulan los libros de texto. Estas preguntas suelen ser muy similares a las que aparecen en las pruebas de evaluación (Gómez Carrasco, Rodríguez Pérez y Miralles Martínez, 2015; Márquez y Roca, 2006), incluidas las estandarizadas (García González y Pérez Martín, 2016). Por lo tanto, esto nos permitiría determinar dónde se pone el foco de la evaluación, a través de su práctica diaria: en el aprendizaje memorístico o en el aplicado.

Por estos y más motivos, actualmente la educación en general (y la científica en particular) se halla en una encrucijada que requiere de un cambio en los procesos de enseñanza-aprendizaje, y dado que los libros de texto son un vector muy importante en el desarrollo del cambio educativo, quisimos analizar qué tipos de preguntas aparecían en los libros de texto de Educación Primaria de las tres últimas leyes educativas. Esta etapa es clave en la introducción de nuevos conceptos y desarrollo de destrezas. Sin olvidar que la erradicación de ideas previas debe iniciarse cuanto antes. En nuestro caso, nos hemos centrado en los temas relacionados con los ecosistemas, ya que es un tema que se trata a lo largo de todos los niveles educativos (Primaria y Secundaria Obligatoria) y su correcto aprendizaje requiere alcanzar los logros reflejados en los estándares de aprendizaje del bloque 3 del Currículum de Educación Primaria (MECD 2014), y además se trata de contenidos de interés para la vida diaria, en el contexto de la sostenibilidad y el medio ambiente.

\section{Objetivos o hipótesis}


El objetivo de investigación general del trabajo fue el análisis de los tipos de preguntas que aparecen en las actividades de diferentes libros de texto de Ciencias naturales de las tres últimas leyes educativas españolas (LOGSE, LOE, LOMCE) para el tema de "los ecosistemas". Con ello, pretendimos valorar qué tipo de aprendizajes se han propuesto en los libros de texto de las diferentes leyes educativas españolas a lo largo del tiempo. Este objetivo general se desglosa en dos objetivos concretos:

- Categorizar los tipos de preguntas de las actividades analizadas en la enseñanza de los ecosistemas en los libros de texto de $5^{\circ}$ y $6^{\circ}$ de Educación Primaria.

- Analizar qué fracción de las preguntas de los libros de texto requieren al estudiante reproducir un contenido presentado (pregunta fáctica o de producto), y cuántas integrar y procesar información para elaborar la respuesta (pregunta de proceso).

\section{Métodos}

En este trabajo, se ha realizado un análisis de contenido sobre las unidades de "Los Ecosistemas", presentes en siete libros de texto de Educación Primaria de las tres últimas leyes educativas españolas que han estado en vigor (LOGSE, LOE, LOMCE). El nivel educativo analizado fue $6^{\circ}$ curso de EP, excepto los libros editados en el marco de la LOMCE, que corresponden a $5^{\circ}$ curso.

\section{Libros de texto}

Para realizar el muestreo elegimos 7 libros de texto de $5^{\circ}$ y $6^{\circ}$ de Educación Primaria de 4 editoriales diferentes (Vicens Vives, Anaya, Luis Vives y SM), editados desde 1999 hasta 2015 en las tres leyes educativas y de uso extendido en centros escolares de Andalucía.

1. Dentro del marco normativo de la LOGSE:

- Casajuana, R., Cruels, E., Escalas, T., García, M., Gatell, C., Ortega, R., Roig, J., (1999), Medio 6 (Andalucía): medio natural, social y cultural, 6 Educación Primaria, 3er ciclo. Sevilla, España. Vicens Vives. En adelante L1.

- Casajuana, R., Cruels, E., Escalas, T., García, M., Gatell, C., Ortega, R., Roig, J., (2001), Medio 6, medio natural, social y cultural, 6 Educación Primaria, 3 ciclo. Sevilla, España. Vicens Vives. En adelante L2.

2. Dentro del marco normativo de la LOE:

- Casajuana, R., Cruells, E., García, M., Gatell, C., Martínez de Murguía, Ma. J., (2006), Tierra, medio natural, social y cultural, 6 Educación Primaria (Andalucía). Sevilla, España. Vicens Vives. En adelante L3.

- Brotons, J.R., Gómez, R., Valbuena, R., (2009), Abre la puerta, Conocimiento del medio, 6 Educación Primaria (Andalucía). Madrid, España. Anaya. En adelante L4.

- Ferreiro, J., Generosa, M., Gómez, L., Otero, A. Ma., Aguilar, E. Mª., Fuente, Mª P., (2009), Proyecto Mundo Agua, conocimiento del medio, 6 Educación Primaria. Sevilla, España. Luis Vives (Edelvives). En adelante L5.

3. Dentro del marco normativo de la LOMCE: 
- González, M., Hidalgo, A., López, S., Navarro, A., Peña, A. y Pueyo, I., (2014). Ciencias de la naturaleza 5 ( $1^{\mathrm{a}}$ ed.). Madrid: SM. En adelante L6.

- Gómez, R. y Valbuena, R. (2015). Ciencias de la naturaleza 5 (1 ${ }^{\text {a }}$ ed.). Madrid: Anaya. En adelante L7.

\section{Procedimiento de la recogida de datos y análisis}

El estudio se centró en el análisis de los tipos de preguntas que aparecen en las actividades. En ocasiones más de una pregunta por actividad, analizándose 257 preguntas, que fueron clasificadas en función del enfoque que requerían para su respuesta siguiendo a Furman, Poenitz y Podestá (2012). Para ello, definimos dos categorías:

- las preguntas de producto o fácticas, que para su respuesta reproducen literalmente conceptos presentados previamente

- las preguntas de proceso, en cuya respuesta se requiere de uso de habilidades científicas para procesar la información.

Asimismo, se crearon subcategorías en función de la demanda precisa que se requería, como se indica en las tablas 1a y $1 \mathrm{~b}$. En ella se explican los criterios de clasificación de las subcategorías relativas al proceso o producto que se demanda. Los datos han sido procesados para su análisis cualitativo (Bardin, 1996) y cuantitativo para el cálculo de las frecuencias de los tipos de preguntas, mediante el programa Microsoft Excel. 
Tabla 1a. Categorización y ejemplos de las preguntas de proceso analizadas en los libros de texto seleccionados.

\begin{tabular}{|c|l|l|}
\hline \multicolumn{3}{|c|}{ TIPOS DE PREGUNTAS DE PROCESO } \\
\hline $\mathbf{N}^{\circ}$ & \multicolumn{1}{|c|}{ SUBCATEGORÍA } & \multicolumn{1}{c|}{ CARACTERÍSTICAS } \\
\hline 1 & De calcular & $\begin{array}{l}\text { Se pide realizar un cálculo y es necesario razonar y } \\
\text { relacionar contenidos para elaborar un } \\
\text { planteamiento para la resolución del problema. }\end{array}$ \\
\hline
\end{tabular}

"En un ecosistema viven 500 especies de seres vivos. Dos quintas partes de esas especies son productoras. También se han contabilizado 20 especies de descomponedores ¿Cuántas especies de consumidores hay?"

$2 \quad \begin{aligned} & \text { De investigación } \\ & \text { experimental }\end{aligned}$

Se pide realizar una experiencia de investigación
(diseño, formulación y el contraste de hipótesis,
recogida y procesamiento de datos, discusión y
elaboración de materiales para presentar los
resultados, etc.).

"Hacemos un terrario. [...]En grupos de cuatro o cinco compañeros, vais a construir un pequeño ecosistema en un terrario [...] Realizamos el terrario. Para construir un terrario debéis seguir los siguientes pasos [...] Organizamos la investigación. Investigad cada uno de los factores de los ecosistemas [...]debes observar el terrario y buscar información en libros, revistas e internet. [...]redacta un informe que contenga los datos más destacados. [...]reuniros todos los miembros del equipo y poned en común el resultado de cada investigación particular. [...] Después, resumid en un informe que expondréis ante la clase, vuestras conclusiones.

Comunicamos los resultados. Para terminar, como en toda investigación, [...] comunicar los resultados. Para ello, el coordinador del equipo leerá los resultados y las conclusiones de la investigación delante de toda la clase. [...]".

\begin{tabular}{|c|c|c|}
\hline 3 & Deducir & $\begin{array}{l}\text { Se pide al alumno que deduzca un posible resultado } \\
\text { a partir de una situación propuesta. }\end{array}$ \\
\hline \multicolumn{3}{|c|}{$\begin{array}{l}\text { “A) ¿Qué les pasaría a los lobos de la imagen si murieran todas las plantas?” } \\
\text { “B) ¿Y qué ocurriría si no hubiera descomponedores como los hongos o las bacterias?” }\end{array}$} \\
\hline 4 & De juicio crítico & $\begin{array}{l}\text { Se pide la opinión razonada del alumno y caben } \\
\text { diferentes razonamientos, puntos de vista e incluso } \\
\text { respuestas. Se requiere del uso de pruebas para } \\
\text { argumentar, construyendo respuestas en las que se } \\
\text { muestra el sentido crítico del aprendiz. }\end{array}$ \\
\hline \multicolumn{3}{|c|}{$\begin{array}{l}\text { "Opina. ¿Crees que esta campaña ayuda a evitar la extinción de especies? ¿Por qué? [...] Propón } \\
\text { otra campaña contra los incendios”." Opina. A Andrea no le gusta que tiren basura en la naturaleza, } \\
\text { pero algunas personas piensan que hay que tirarla en algún sitio. ¿A ti, qué te parece?”. }\end{array}$} \\
\hline 5 & $\begin{array}{l}\text { De razonar/explicar la } \\
\text { respuesta o justificarla con } \\
\text { argumentos }\end{array}$ & $\begin{array}{l}\text { Se pide una explicación razonada con pruebas y } \\
\text { elaboradas con sus palabras evitando la } \\
\text { reproducción literal del libro de texto. Aunque caben } \\
\text { diferentes razonamientos, la solución buscada es } \\
\text { única. }\end{array}$ \\
\hline \multicolumn{3}{|c|}{$\begin{array}{l}\text { "1. ¿Para qué les sirven a los seres vivos las adaptaciones que presentan? 2. Justifica tu opinión. } \\
\text { ¿Crees que las alas de un pájaro son una adaptación?”. “QQué ocurriría si se talara el bosque y se } \\
\text { dedicara a pastos? Razona tu respuesta”. }\end{array}$} \\
\hline 6 & dibujar/actividades & $\begin{array}{l}\text { Se requiere del uso de actividades plásticas en las } \\
\text { que no se requiere de una simple reproducción de } \\
\text { un dibujo o modelo ejemplificado en el libro, sino en } \\
\text { las que el estudiante desarrolle una propuesta } \\
\text { personalizada y que exija pensar qué quiere crear. }\end{array}$ \\
\hline \multicolumn{3}{|c|}{ “Dibuja el planeta desnudo y complétalo con todo lo necesario para hacerlo habitable”. } \\
\hline 7 & De puesta en común & $\begin{array}{l}\text { Se reflexiona en grupo y contraponiendo propuestas } \\
\text { para resolver una situación propuesta. }\end{array}$ \\
\hline \multicolumn{3}{|c|}{$\begin{array}{l}\text { "Forma, con otros compañeros y compañeras, un equipo de trabajo en el que debatáis qué podéis } \\
\text { hacer para contribuir al desarrollo sostenible y en el que preparéis un mural que resuma vuestras } \\
\text { conclusiones". }\end{array}$} \\
\hline
\end{tabular}


Tabla 1b. Categorización y ejemplos de las preguntas fácticas analizadas en los libros de texto seleccionados.

\begin{tabular}{|c|c|c|}
\hline \multicolumn{3}{|c|}{ TIPOS DE PREGUNTAS FÁCTICAS } \\
\hline $\mathbf{N}^{\circ}$ & SUBCATEGORÍA & CARACTERÍSTICAS \\
\hline 1 & $\begin{array}{l}\text { Elaborar informes en soporte } \\
\text { digital }\end{array}$ & $\begin{array}{l}\text { Se trata de preguntas que proponen la elaboración } \\
\text { de documentos en formato digital (procesador de } \\
\text { textos) con reproducción literal de información y en } \\
\text { un formato muy definido. }\end{array}$ \\
\hline \multicolumn{3}{|c|}{$\begin{array}{l}\text { "Copia la lectura sobre Dian Fossey: Usa la fuente Comic Sans MS y el tamaño 12. Pon en negrilla } \\
\text { Dian Fossey, en cursiva todas las fechas y subraya la palabra gorilas. Por último, guarda el documentc } \\
\text { en tu carpeta personal". }\end{array}$} \\
\hline 2 & $\begin{array}{llr}\text { Identificar } & \text { y } & \text { seleccionar } \\
\text { palabras } & 0 & \text { conceptos } \\
\text { presentes en el libro de texto }\end{array}$ & $\begin{array}{l}\text { Proponen la búsqueda de palabras, ideas y/o } \\
\text { información tanto en el propio libro como en otras } \\
\text { fuentes y su reproducción literal en el documento } \\
\text { que se entrega. }\end{array}$ \\
\hline \multicolumn{3}{|c|}{$\begin{array}{l}\text { "Además del caso de los piojos y los mamíferos, en el texto se menciona otra relación de parasitismo. } \\
\text { ¿Cuál es?" }\end{array}$} \\
\hline 3 & Comparar/Contrastar & $\begin{array}{l}\text { Se requiere que comparen o contrasten } \\
\text { informaciones prácticamente literales previamente } \\
\text { dadas por el propio libro de texto. }\end{array}$ \\
\hline \multicolumn{3}{|c|}{$\begin{array}{l}\text { "Observa las fotografías de estos tres ecosistemas. } \\
\text { 1. Di qué ecosistemas son y ordénalos en tu cuaderno según su tamaño. } \\
\text { 2. ¿En qué lugar de la Tierra te parece que podríamos encontrarlos? } \\
\text { 3. Indica qué condiciones ambientales se dan en cada uno y compáralas. } \\
\text { 4. ¿Qué seres vivos pueden vivir en cada uno?". }\end{array}$} \\
\hline 4 & Completar huecos & $\begin{array}{l}\text { Se pide que completen frases } \circ \text { esquemas } \\
\text { incompletos donde faltan palabras. }\end{array}$ \\
\hline \multicolumn{3}{|c|}{$\begin{array}{l}\text { "Copia y completa el resumen. } \\
\text { La biosfera es la zona de la Tierra en la que podemos encontrar__, y se divide en partes más } \\
\text { pequeñas llamadas_. Los ecosistemas están formados por el___ lo___ las__ } \\
\text { que se dan entre ellos". }\end{array}$} \\
\hline 5 & Definir/describir/redactar & $\begin{array}{l}\text { Consisten en la definición de un concepto que } \\
\text { normalmente está mencionado en las páginas } \\
\text { anteriores, por lo que resulta una reproducción de lo } \\
\text { leído. }\end{array}$ \\
\hline \multicolumn{3}{|c|}{$\begin{array}{l}\text { "Describe tres acciones para reducir la contaminación" } \\
\text { "Define el término comunidad biológica e indica en qué se diferencia del concepto población biológica". }\end{array}$} \\
\hline 6 & Sintetizar y esquematizar & $\begin{array}{l}\text { Se pide que hagan esquemas de algún apartado del } \\
\text { tema. }\end{array}$ \\
\hline \multicolumn{3}{|c|}{$\begin{array}{l}\text { "Haz un esquema en el que clasifiques y resuman las relaciones que has estudiado en esta página, } \\
\text { añadiendo algún ejemplo". }\end{array}$} \\
\hline 7 & Enumerar listas de casos & $\begin{array}{l}\text { Se pide la identificación de conceptos del tema, así } \\
\text { como citarlos, elaborar listas o clasificarlos. }\end{array}$ \\
\hline \multicolumn{3}{|c|}{ "Haz una lista de los seres vivos que aparecen en la red trófica del mar". } \\
\hline 8 & Estimar cálculo & $\begin{array}{l}\text { Se pide la resolución de un problema cuyo } \\
\text { planteamiento se considera explícito y sugiere un } \\
\text { cálculo directo. }\end{array}$ \\
\hline
\end{tabular}

Finalmente, se calcularon las frecuencias de preguntas de cada categoría y/o subcategoría, y se utilizaron para analizar las tendencias de los tipos de preguntas que los estudiantes han tenido que resolver en Educación Primaria a lo largo del tiempo en las tres leyes educativas. 


\section{Resultados y Discusión}

De las 257 preguntas analizadas, 184 son de tipo fáctico y sólo 73 de proceso. En este sentido, nuestro estudio sugiere que las preguntas de los libros de texto de Ciencias naturales han promovido aprendizajes memorísticos, ya que el $71,6 \%$ de las preguntas analizadas fueron de este tipo. Esta circunstancia no es nueva, ni exclusiva de España (Furman et al., 2012; García González y Pérez Martín, 2016), ni tampoco de esta área de conocimiento, ya que han existen estudios similares con los mismos resultados sobre preguntas de examen de Ciencias sociales en EP (Miralles, Gómez Carrasco y Sánchez Ibáñez, 2014) o Física en enseñanzas medias (Alonso, Gil y Martínez Torregrosa, 1992), entre otros. Sin embargo, en el caso de los ecosistemas resulta preocupante porque la temática que aborda tiene fuerte relación con la Ecología y la Educación Ambiental. Un conjunto de conocimientos, calificados de temas transversales y que resultan de gran interés para el pleno desarrollo como adultos científicamente alfabetizados, en un mundo que debe promover la sostenibilidad medioambiental (Cebrián y Junyent, 2014).

Hay que considerar que cada vez son más los contenidos en Ecología que suelen presentarse como controversias socio-científicas, lo que exige al aprendiz el procesamiento de informaciones para generar respuestas que promuevan el desarrollo de un pensamiento crítico (Pérez Martín y Bravo Torija, 2018). Sin embargo, la evaluación de contenidos memorizados sin procesar promueve el aprendizaje de dogmas y evita la controversia, lo que es frecuente en la presentación de contenidos en los libros de texto (Braga Blanco y Belver Domínguez, 2016).

Si consideramos cómo se han presentado las preguntas en los libros de texto a lo largo de las tres últimas legislaciones educativas españolas, los resultados muestran que siempre han propuesto mayoritariamente preguntas de tipo fáctico. En la figura 1, se puede ver que las editoriales de la etapa LOGSE más del $90 \%$ de las preguntas eran de tipo fáctico. Sin embargo, con los sucesivos cambios legislativos, que sobre el papel promovían aprendizajes más competenciales, esta tendencia se ha ido modificando, incrementando el número de preguntas que promueven la argumentación y el uso de pruebas como las preguntas de razonar o justificar la respuesta y las de juicio crítico. Aunque la transición hacia este tipo de cuestiones ha sido paulatina, no es claramente evidente hasta la actual ley educativa, donde se aprecia que los contenidos que se tratan en el bloque de los ecosistemas son evaluados con actividades de ambos tipos casi por igual (Figura 1). Con todo ello, se ve que las editoriales han promovido cambios en los tipos de preguntas marcando una tendencia de reducción de preguntas fácticas y un aumento en las de proceso, llegando casi a equipararse en las ediciones más actuales (LOMCE). A pesar de ello, cabe señalar que no deberíamos responsabilizar exclusivamente a la LOMCE, ya que la necesidad de aprendizajes competenciales ya fue definida en la LOE (García Fernández, Mateos Jiménez, y Romo-Pérez 2017). Sin embargo, no ha sido hasta el cambio de ley y su nuevo desarrollo, cuando las editoriales han reconducido su línea pedagógica, y han cambiado sus preguntas, encaminando, aparentemente, a los estudiantes hacia aprendizajes críticos a través de las preguntas de proceso. 


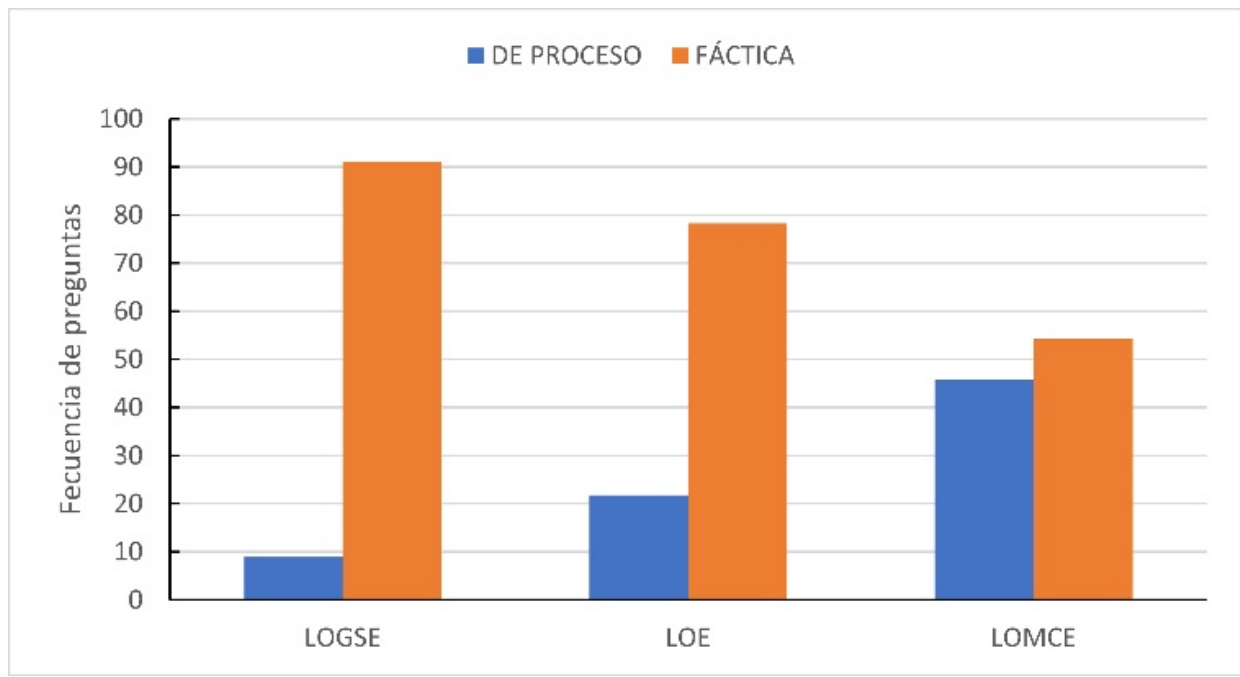

Figura 1. Porcentaje de preguntas de proceso y fácticas de los libros de texto de Ciencias naturales en Educación Primaria para el tema de los ecosistemas en los distintos periodos legislativos en España.

El recuento y la categorización pormenorizada de los tipos de preguntas analizadas se presentan en la tabla 2 para las preguntas de proceso y, en la tabla 3 para las preguntas fácticas. Todas ellas se clasificaron en función de la tarea encomendada en la pregunta, como detallamos en la metodología, donde presentamos un ejemplo de cada tipo (Tablas $1 \mathrm{a}$ y $1 \mathrm{~b})$.

En la tabla 2 se observa que los dos tipos de preguntas de proceso más frecuentes son las que piden razonar, explicar o justificar la respuesta $(57,5 \%)$, seguidas de las reflexionar u opinar (juicio crítico) sobre algún aspecto de los contenidos presentados $(26,0 \%)$. En esta misma línea, podemos decir que las preguntas de calcular, realizar experiencias investigables o realizar puestas en común son minoritarias y sólo se encuentran en libros de texto de EP del periodo LOE. Esta carencia ya había sido detectada por otros autores tanto en EP (Verde Romera, Caballero Caballero y Pablos Miguel, 2017) como en Educación Secundaria (Occelli y Valeiras, 2013). Por lo tanto, esta situación pone de manifiesto la necesidad de introducir actividades prácticas en la formación de los estudiantes para promover sus destrezas científicas (Martí, 2012; Occelli y Valeiras, 2013). Por último, queremos destacar un último tipo de preguntas de proceso que requieren de la elaboración de materiales artísticos en el contexto de la asignatura de Educación Artística. Este tipo de preguntas promueven destrezas comunicativas en el contexto de las ciencias (Pro, 2013), tal y como analizaron García González y Pérez Martín (2016).

En la tabla 1b se muestran algunos ejemplos de las categorías de preguntas fácticas encontradas, y en la tabla 3 se muestran las frecuencias en valores absolutos. Las tres categorías más encontradas en nuestro estudio son i) las que piden enumerar listas de casos $(33,2 \%)$, ii) las de definir, describir o redactar cuestiones que se presentan en las páginas anteriores y se reproducen copiándose literalmente $(22,2 \%)$ y iii) las que piden identificar y seleccionar palabras o ideas del libro de texto y reproducirlas literalmente $(21,2 \%)$. Cabe destacar que en esta categoría se han cuantificado también preguntas reproductivas del conocimiento a estudiar, como las de completar huecos, comparar o esquematizar. Por último, hay que reseñar que son muy minoritarias las de copiar información en soporte digital y de estimar cálculos. 
Tabla 2. Clasificación de las demandas requeridas en las preguntas de proceso analizadas en los libros de texto.

\begin{tabular}{|c|c|c|c|c|c|c|c|c|c|}
\hline \multicolumn{10}{|c|}{ TIPOS DE PREGUNTAS DE PROCESO } \\
\hline \multirow{2}{*}{\multicolumn{2}{|c|}{ CATEGORÍA }} & \multicolumn{8}{|c|}{ LIBRO } \\
\hline & & \multicolumn{2}{|c|}{ LOGSE } & \multicolumn{3}{|c|}{ LOE } & \multicolumn{2}{|c|}{ LOMCE } & \multirow{2}{*}{ Total } \\
\hline $\mathbf{N}^{\circ}$ & PREGUNTAS & L1 & L2 & L3 & L4 & L5 & L6 & L7 & \\
\hline 1 & De calcular & 0 & 0 & 0 & 0 & 0 & 0 & 1 & 1 \\
\hline 2 & De investigación experimental & 0 & 0 & 0 & 1 & 1 & 0 & 0 & 2 \\
\hline 3 & Deducir & 0 & 0 & 0 & 0 & 0 & 0 & 2 & 2 \\
\hline 4 & De juicio crítico & 0 & 1 & 0 & 6 & 1 & 10 & 1 & 19 \\
\hline 5 & $\begin{array}{l}\text { Razonar/explicar la respuesta } \\
\text { o justificarla con argumentos }\end{array}$ & 1 & 2 & 3 & 7 & 5 & 17 & 7 & 42 \\
\hline 6 & Dibujar/actividades plásticas & 0 & 0 & 0 & 0 & 1 & 4 & 1 & 6 \\
\hline 7 & Puesta en común, debate & 0 & 0 & 0 & 0 & 0 & 0 & 1 & 1 \\
\hline \multicolumn{2}{|r|}{ Total preguntas de Proceso } & 1 & 3 & 3 & 14 & 8 & 31 & 13 & 73 \\
\hline
\end{tabular}

En nuestra opinión, es razonable que este tipo de preguntas aparezcan en la evaluación. Sin embargo, si comparamos las categorías más numerosas en las tablas 2 y 3 veremos que el total de los tres tipos de preguntas fácticas mayoritarias (127) representan casi el doble que las de proceso (67). Por lo que, con este tipo de programación de la evaluación, se obvia la demanda de formación crítica para una alfabetización científica que promueva la Justicia Social en la toma de decisiones (Murillo y Hernández Castilla, 2011), conduciéndonos a una escuela poco democrática.

Tabla 3. Clasificación de las demandas requeridas en las preguntas fácticas analizadas en los libros de texto.

\begin{tabular}{|c|c|c|c|c|c|c|c|c|c|}
\hline \multicolumn{10}{|c|}{ TIPOS DE PREGUNTAS FÁCTICAS } \\
\hline \multirow{2}{*}{\multicolumn{2}{|c|}{ CATEGORÍA }} & \multicolumn{8}{|c|}{ LIBRO } \\
\hline & & \multicolumn{2}{|c|}{ LOGSE } & \multicolumn{3}{|c|}{ LOE } & \multicolumn{2}{|c|}{ LOMCE } & \multirow{2}{*}{ Total } \\
\hline $\mathbf{N}^{\circ}$ & PREGUNTAS & L1 & L2 & L3 & L4 & L5 & L6 & L7 & \\
\hline 1 & $\begin{array}{l}\text { Elaborar informes en } \\
\text { soporte digital }\end{array}$ & 0 & 0 & 0 & 0 & 1 & 0 & 0 & 1 \\
\hline 2 & $\begin{array}{l}\text { Identificar y seleccionar } \\
\text { palabras o conceptos } \\
\text { presentes en el libro de } \\
\text { texto }\end{array}$ & 4 & 5 & 4 & 7 & 8 & 5 & 6 & 39 \\
\hline 3 & Comparar/Contrastar & 3 & 2 & 3 & 2 & 2 & 1 & 0 & 13 \\
\hline 4 & Completar huecos & 0 & 3 & 1 & 0 & 3 & 2 & 1 & 10 \\
\hline 5 & Definir/describir/redactar & 5 & 0 & 3 & 15 & 6 & 2 & 10 & 41 \\
\hline 6 & Sintetizar y esquematizar & 1 & 2 & 2 & 4 & 1 & 1 & 5 & 16 \\
\hline 7 & Enumerar listas de casos & 7 & 8 & 9 & 7 & 12 & 10 & 8 & 61 \\
\hline 8 & Estimar cálculos & 0 & 1 & 0 & 0 & 0 & 0 & 1 & 2 \\
\hline \multicolumn{2}{|r|}{ Total Preguntas Fácticas } & 20 & 21 & 22 & 35 & 33 & 22 & 31 & 184 \\
\hline
\end{tabular}

En este punto cabe destacar, que muchos libros presentan preguntas que a priori pueden parecer preguntas de proceso, pero cuando se analizan con detalle se pude 
apreciar que son preguntas fácticas. Un ejemplo de ello se puede ver en el libro L2, donde existe un apartado de actividades titulado "Razona y Contesta", aunque en realidad la actividad promueve la copia literal de definiciones tras proceder como si de un cuestionario de verdadero o falso se tratase.

Razona y contesta. Copia en tu cuaderno las frases verdaderas:

1. La comunidad biológica de un ecosistema es el conjunto de todos los organismos que viven en él.

2. Las diferentes poblaciones de un ecosistema están formadas por el mismo número de individuos.

\section{La hibernación es una adaptación de algunos mamíferos a la temperatura.}

4. Los consumidores primarios son los animales que toman alimentos de origen vegetal, es decir, herbívoros.

Otro ejemplo se puede ver en un libro de texto más actual L7. Tiene un bloque titulado "Comprende, piensa, investiga". Sin embargo, las preguntas planteadas se responden mirando la imagen a la que hacen alusión, pues les piden buscar los seres vivos que aparecen en ella.

\section{COMPRENDE, PIENSA, INVESTIGA}

1. Haced una lista con los seres vivos que se ven en la imagen del bosque. Haced otra lista con los otros seres vivos que podríais encontrar en un bosque observando con atención.

2. Indicad dónde hay bellotas en la imagen y qué árboles las producen.

De modo que, aunque los títulos de las actividades parecen indicar que van a plantear preguntas de proceso, en las que se promueve el razonamiento, no siempre es el caso.

Según nuestros datos, en el caso de las preguntas de proceso (Tabla 2), el número de preguntas de proceso publicadas por las editoriales en los libros de texto sobre juicio crítico, razonar y justificar con argumentos y trabajar las ciencias desde las artes plásticas han aumentado en los últimos años. Sin embargo, preguntas de calcular, de deducir, de puestas en común y de realización de actividades experimentales no presentan un volumen suficiente para considerar que sus tendencias han cambiado entre las legislaciones. Con nuestros datos, podemos decir que la variedad de tipos de preguntas de proceso se ha incrementado con las sucesivas legislaciones. Las preguntas de proceso en los libros de texto LOGSE eran escasas, y las que aparecen son todas de juicio crítico y de razonar; con la LOE hay cuatro categorías de preguntas, y con la LOMCE seis. Sin embargo, nuestros resultados indican que muchas de ellas están lejos de ser preguntas investigables o de pensar.

Por el contrario, en el caso de las preguntas fácticas (Tabla 3), existen un conjunto de ellas que no han variado a lo largo del tiempo como son las de completar huecos, definir, esquematizar y de enumerar listados de casos. Lo cual parece sugerir que son actividades que las editoriales consideran valiosas para el aprendizaje de los estudiantes. En este sentido, cabe reseñar que hay dos que han disminuido su número como son las de buscar información y las de comparar o contrastar. Al igual que ocurría en el caso de las preguntas de proceso, hay actividades que no se pueden analizar en 
este sentido por ser anecdóticas en los libros de texto analizados (elaborar informes y estimar cálculos). En conjunto, estos resultados ponen de manifiesto que el diseño de las evaluaciones es muy conservador, promoviendo poco los aprendizajes mediados por el razonamiento.

\section{Continuidad de la línea editorial}

Por último, quisimos analizar si las editoriales realizan cambios en los libros de texto de ediciones consecutivas. Para ello, analizamos dos editoriales en la transición entre dos marcos legislativos consecutivos: Vicens Vives en LOGSE (L1 y L2) y LOE (L3) y Anaya en LOE (L4) y LOMCE (L7).

A pesar de las limitaciones del estudio, en el caso de Vicens Vives (L1, L2, L3), podemos observar en la tabla 2 cómo las preguntas de razonar y justificar con argumentos se incrementan en una más en cada edición. El resto de categorías de preguntas de proceso no aparecen representadas en los libros, excepto una de juicio crítico que aparece exclusivamente en L2. Llama la atención que son las únicas preguntas de proceso que aparecen en estos libros sobre este tema. El caso de las preguntas fácticas (Tabla 4) es diferente ya que, al ser mayores en número, podemos observar ciertas tendencias. En general, observamos que el número de preguntas de cada tipo es prácticamente constante en las tres ediciones para todas las preguntas, excepto las de completar huecos y las de definir que muestran pequeños vaivenes. Lo que pone de relevancia que las preguntas que se formulan en ediciones sucesivas de libros de texto son muy constantes en el tiempo (Braga Blanco y Belver Domínguez, 2016; Carmen, 2010; Carmen y Jiménez-Aleixandre, 2010), aunque transcurra más de un lustro entre ediciones.

\begin{tabular}{|c|c|c|c|c|}
\hline \multicolumn{5}{|c|}{$\begin{array}{l}\text { Tabla 4. Análisis de la continuidad editorial entre LOGSE y LOE en las } \\
\text { preguntas fácticas propuestas por Vicens Vives. }\end{array}$} \\
\hline \multicolumn{5}{|c|}{ TIPOS DE PREGUNTAS FÁCTICAS (Vicens Vives) } \\
\hline \multirow{2}{*}{\multicolumn{2}{|c|}{ CATEGORÍA }} & \multicolumn{3}{|c|}{ LIBRO } \\
\hline & & \multicolumn{2}{|c|}{ LOGSE } & \multirow{2}{*}{$\frac{\text { LOE }}{\text { L3 }}$} \\
\hline $\mathbf{N}^{\circ}$ & PREGUNTAS & L1 & L2 & \\
\hline 1 & Elaborar informes en soporte digital & 0 & 0 & 0 \\
\hline 2 & $\begin{array}{lll}\text { Identificar y seleccionar palabras } & 0 \\
\text { conceptos presentes en el libro de texto } & \end{array}$ & 4 & 5 & 4 \\
\hline 3 & Comparar/Contrastar & 3 & 2 & 3 \\
\hline 4 & Completar huecos & 0 & 3 & 1 \\
\hline 5 & Definir/describir/redactar & 5 & 0 & 3 \\
\hline 6 & Sintetizar y esquematizar & 1 & 2 & 2 \\
\hline 7 & Enumerar listas de casos & 7 & 8 & 9 \\
\hline 8 & Estimar cálculos & 0 & 1 & 0 \\
\hline & Total Preguntas Fácticas & 20 & 21 & 22 \\
\hline
\end{tabular}

En el caso del segundo caso de editorial analizado (Anaya, L4 y L7, Tablas 2 y 5), observamos que el número total de preguntas de una edición a la siguiente se reduce en 5,1 de proceso y 4 fácticas. Por lo que el cambio que se observa es más en la reducción de la evaluación que en el tipo de preguntas realizadas. En un análisis por separado, podemos ver que las preguntas de proceso mantienen un número total prácticamente idéntico (LOE 14 frente a LOMCE 13). Además, se detectan variaciones muy pequeñas en el número de preguntas categorizadas en el estudio. Ejemplos de ello son las preguntas de cálculo, de investigación experimental, de deducir, de uso de 
actividades plásticas y de puestas en común. En estos casos hay categorías de preguntas que aparecen o desaparecen de LOE a LOMCE. Llama la atención el mantenimiento del número de preguntas de razonar y justificar con argumentos, pero también la reducción drástica de las preguntas de juicio crítico que pasan de 6 en LOE a 1 en LOMCE. Lo que podemos interpretar como un ejemplo de que en los libros de ciencias naturales se prefieren actividades de evaluación que no cuestionan la complejidad de los contenidos, promoviendo una enseñanza de las ciencias de tipo cerrado en la que los estudiantes no participan y los ven ajenos a ellos (Furman, 2013), lo que fomenta su desafección por las ciencias (Solbes, 2011).

En el caso de las preguntas fácticas, destacamos que de las ocho categorías de la tabla 5 , dos de ellas sufren reducciones del número de preguntas en la edición LOMCE respecto de la edición LOE: las de comparar que desaparecen; y las de definir que se reducen en un $33 \%$. Hay otras tres categorías que se podrían considerar constantes en número (identificar y seleccionar palabras, esquematizar y enumerar listas de casos), ya que mantienen el mismo número de preguntas en ambas ediciones. Por último, hay dos categorías de preguntas que aparecen en la edición LOMCE y que no estaban en LOE: completar huecos y estimar cálculos, con un caso cada una.

\begin{tabular}{|c|c|c|c|}
\hline \multicolumn{4}{|c|}{$\begin{array}{l}\text { Tabla 5. Análisis de la continuidad editorial entre LOE y LOMCE en } \\
\text { las preguntas fácticas propuestas por Anaya. }\end{array}$} \\
\hline \multicolumn{4}{|c|}{ TIPOS DE PREGUNTAS FÁCTICAS (Anaya) } \\
\hline \multicolumn{2}{|r|}{ CATEGORÍA } & \multicolumn{2}{|c|}{ LIBRO } \\
\hline & & \multirow{2}{*}{$\frac{\text { LOE }}{\text { L4 }}$} & \multirow{2}{*}{$\begin{array}{c}\text { LOMCE } \\
\text { L7 }\end{array}$} \\
\hline $\mathbf{N}^{\circ}$ & PREGUNTAS & & \\
\hline 1 & Elaborar informes en soporte digital & 0 & 0 \\
\hline 2 & $\begin{array}{l}\text { Identificar y seleccionar palabras o } \\
\text { conceptos presentes en el libro de texto }\end{array}$ & 7 & 6 \\
\hline 3 & Comparar/Contrastar & 2 & 0 \\
\hline 4 & Completar huecos & 0 & 1 \\
\hline 5 & Definir/describir/redactar & 15 & 10 \\
\hline 6 & Sintetizar y esquematizar & 4 & 5 \\
\hline 7 & Enumerar listas de casos & 7 & 8 \\
\hline 8 & Estimar cálculos & 0 & 1 \\
\hline & Total Preguntas Fácticas & 35 & 31 \\
\hline
\end{tabular}

analizar si se repetían las mismas preguntas en diferentes ediciones, lo que ocurre en el caso de Vicens Vives (L1: 1999, L2: 2001 y L3: 2006) y Anaya (L4: 2009 y L7: 2015) y representamos con estos ejemplos.

L1: Razona y contesta. 1. ¿Qué diferencia existe entre una comunidad biológica y un ecosistema?

L2: HAZ MEMORIA. Lee el texto del primer apartado y recuerda el significado de las palabras destacadas en negrita: comunidad biológica y población.

\section{L3: 4. ¿Qué diferencia existe entre una comunidad biológica y un ecosistema?}

En el caso de Anaya ( $L 4$ y L7), también se repiten actividades de forma literal o casi literal, pero siendo en síntesis lo mismo, como se muestra en los siguientes ejemplos: 
L4: 1. Nombra cinco recursos naturales que los seres humanos utilizamos en nuestras actividades.

\section{L7: Cita algunos recursos naturales que uses en tu casa.}

En otro ejemplo, se pide que hagan un esquema de las relaciones estudiadas ecológicas en las páginas donde está propuesta la actividad, en ambas ediciones las imágenes son similares, en concreto se refiere a las relaciones de las abejas, las garrapatas y las orquídeas, y usan incluso la misma fotografía.

L4: 1. Organiza en un esquema los tipos de relaciones que se mencionan en estas páginas.

L7: haz un esquema en el que clasifiques y resumas las relaciones que has estudiando en esta página, añadiendo algún ejemplo.

La repetición de preguntas en la evaluación de un contenido es frecuente según estudios realizados por diferentes autores (Carmen 2010; Carmen y Jiménez-Aleixandre 2010), y per se no tiene por qué ser considerado inadecuado para la formación de los aprendices. Sin embargo, los cambios legislativos que se promovieron en la LOE y posteriormente en la LOMCE deberían encaminar, al menos, a equilibrar las evaluaciones hacia preguntas más de indagación, más experimentales y sobre todo que requieran del pensamiento aplicado de los estudiantes, preguntas que se catalogarían de proceso, pero esto no es lo que indican los datos de nuestro estudio.

\section{Conclusiones}

Los libros de texto de las etapas LOGSE y LOE presentaban mayor número de preguntas fácticas que de proceso, por lo que se considera que los aprendizajes promovidos en Educación Primaria eran memorísticos. Con el paso a LOMCE, los libros de texto han modificado sus preguntas de evaluación incorporando preguntas de proceso y poniendo casi al mismo nivel ambos tipos de preguntas, lo que permite un proceso de enseñanza-aprendizaje más equilibrado entre memorización y aplicación de conocimientos. Sin embargo, aún son infrecuentes preguntas que exijan pensar e investigar a los estudiantes.

A pesar de ello, los libros de texto han cambiado poco su forma de formular preguntas entre ediciones consecutivas de leyes educativas diferentes, repitiendo incluso preguntas literales. También presentan títulos para las preguntas que no son veraces, (Comprende, piensa e investiga"), ya que sus enunciados no exigen de estas acciones.

Por último, cabe destacar que, ante las muchas propuestas de análisis de actividades, el análisis de preguntas categorizado de forma sencilla de nuestro estudio ("fácticas" frente a "de proceso"), simplifica el análisis y permite una interpretación objetiva del espíritu del aprendizaje que promueven las herramientas de evaluación.

Estos resultados muestran la necesidad de profundizar de forma sistemática en la forma de preguntar en las aulas, en concreto de los libros de texto. Mejorando la formulación de preguntas se puede mejorar el interés por las ciencias, mostrándolas como una construcción humana de la que todo ciudadano debe ser partícipe. Esta necesidad de mejora en la formulación de preguntas debe poner en contacto para trabajar en la misma dirección a maestros, investigadores en Didáctica de las Ciencias experimentales y profesionales de la industria editorial. 


\section{Referencias}

Alonso Sánchez, M. Gill D. y Martínez Torregrosa, J. (1992). Los exámenes de Física en la enseñanza por transmisión y en la enseñanza por investigación. Enseñanza de las Ciencias, 10(2), 127-138

Altbach, P. y Kelly, G. (1988). Textbooks in the Third World. Policy, content and context. New York: Garland.

Andersson, K., Gullberg, A. (2014). What is science in preschool and what do teachers have to know to empower children? Cultural Studies of Science Education 9(2), 275-296.

Apple, MW. (1993). El libro de texto y la política cultural. Revista de Educación, 301, 109-126.

Area, M. (2009). Introducción a la tecnología educativa. Universidad de La Laguna: Creative Commons. Recuperado de https://manarea.webs.ull.es/wpcontent/uploads/2010/06/ebookte.pdf

Arriassecq, I. y Greca, I. (2004). Enseñanza de la teoría de la relatividad especial en el ciclo polimodal: dificultades manifestadas por los docentes y textos de uso habitual. Revista Electrónica de Enseñanza de las Ciencias, 3(2), 211227.

Asociación Nacional de Editores de Libros y Material de Enseñanza (ANELE). (2013). Informe sobre la edición de libros de texto en España. Recuperado de https://goo.gl/ezWErs

Bardin, L. (1996). El análisis del contenido (2ª ed.). Madrid: Akal.

Beas, M. (1999).. Los libros de texto y las Comunidades Autónomas: una pesada Torre de Babel. Revista Complutense de Educación, 10(2), 29-52.

Borges Fernandes, IM., Pires, DM. y Delgado-Iglesias, J. (2017). Las relaciones entre Ciencia, Tecnología, Sociedad y Ambiente, en los libros de texto de Educación Primaria: Un estudio comparativo entre Portugal y España, antes de las últimas reformas educativas. Revista Eureka sobre Enseñanza y Divulgación de las Ciencias, 14(1), 54-68.

Braga Blanco, GM. y Belver Domínguez, JL. (2016). El análisis de libros de texto: una estrategia metodológica en la formación de los profesionales de la educación. Revista Complutense de Educación, 27(1), 199-218.

Calvo Pascual, MA. y Martín Sánchez, M. (2005). Análisis de la adaptación de los libros de texto de ESO al currículo oficial, en el campo de la Química. Enseñanza de las Ciencias, 23(1), 1732.

Cantó, J., Pro, A. y Solbes, J. (2016). ¿Qué ciencias se enseñan y cómo se hace en las aulas de educación infantil? La visión de los maestros en formación inicial. Enseñanza de las Ciencias, $34(3), 25-50$.

Carmen, L. (2010). Los materiales de desarrollo curricular: un cambio imprescindible. Investigación en la Escuela, 72, 53-60.

Carmen, LM. y Jiménez Aleixandre, MP. (2010). Los libros de texto: un recurso flexible. Alambique. Didáctica de las Ciencias Experimentales, 17(66), 48-55.
Cebrián, G. y Junyent, M. (2014). Competencias profesionales en Educación para la Sostenibilidad: un estudio exploratorio de la visión de futuros maestros. Enseñanza de las Ciencias, 32(1), $29-49$

Certad Villarroel, PA. (2013). Análisis del texto escolar de ciencias naturales desde la transdisciplinariedad. Revista de la SEECI, 31, 52-69.

Concari, SB. y Giorgi, SM. (2000). Los Problemas Resueltos en Textos Universitarios de Física. Enseñanza de las Ciencias, 18(3), 381-390.

Costa Amaral, CL., Da Silva Xavier, E. y Maciel, MD. (2009). Abordagem das relações Ciência/Tecnologia/Sociedade nos conteúdos e funções orgânicas em livros didáticos de Química do ensino médio. Investigações em Ensino de Ciências, 4(1), 101-114.

Ferreiro, G. y Occelli, M. (2008). Análisis del abordaje de la respiración celular en textos escolares para el Ciclo Básico Unificado. Revista Electrónica de Enseñanza de las Ciencias, 7(2), 387-398

Furman, M. (2013). Enseñar ciencias naturales: Lejos del dogma y cerca de la aventura. Ruta Maestra, 5, 48-54.

Furman, M., Poenitz, MV. y Podestá, ME. (2012). La evaluación en la formación de los profesores de Ciencias. Praxis \& Saber, $3(6), 165-189$

García, DP. (2001). Los usos de los libros de texto en la práctica docente cotidiana de tercero y cuarto de primaria: un estudio cualitativo. México: Departamento de Investigaciones Educativas Cinvestav.

García-Carmona, A., Cruz-Guzmán, M. y Criado, AM. (2014). ¿Qué hacías para aprobar los exámenes de Ciencias, qué aprendiste y qué cambiarías? Investigación en la Escuela, 84, 31-46.

García-Rodeja Gayoso, I. (1997). ¿Qué propuestas de actividades hacen los libros de primaria? Alambique. Didáctica de las Ciencias Experimentales, 11, 35-43.

García Fernández, B., Mateos Jiménez, A. y Romo-Pérez, V. (2017). Construcción y validación de un instrumento para identificar las percepciones de los docentes de Ciencias sobre el modelo de enseñanza por competencias. Estudios pedagógicos (Valdivia), 43(1), 139-156.

García-González, S. y Pérez-Martín, JM. (2016). Enseñanza de las Ciencias naturales en Educación Primaria a través de cuentos y preguntas mediadoras. Revista Internacional de Investigación e Innovación en Didáctica de las Humanidades y las Ciencias, 3, 101-122.

Gómez Carrasco, CJ., Rodríguez Pérez, RA. y Miralles Martínez, P. (2015). La enseñanza de la Historia en educación primaria y la construcción de una narrativa nacional: Un estudio sobre exámenes y libros de texto en España. Perfiles educativos, $37(150), 20-38$

Instituto Nacional de Evaluación Educativa (INEE). (2017). PIRLS 2016: Estudio internacional de progreso en comprensión lectora. IEA. Informe español. España: MECD. Recuperado de https://bit.ly/2LHxUG1 
López-Manjón, A. y Postigo, Y. (2016). ¿Qué libro de texto elegir? La competencia visual en las actividades con imágenes. Revista Eureka sobre Enseñanza y Divulgación de las Ciencias, 13(1), 84-101.

López-Valentín, DM. y Guerra-Ramos, MT. (2013). Análisis de las actividades de aprendizaje incluidas en libros de texto de ciencias naturales para educación primaria utilizados en México. Enseñanza de las Ciencias, 31(2), 173-191.

Márquez, C., Bonil, J. y Pujol, RM. (2005). Las preguntas mediadoras como recursos para favorecer la construcción de modelos científicos complejos. Enseñanza de las Ciencias, Número extra, 1-5.

Márquez, C. y Roca, M. (2006). Plantear preguntas: un punto de partida para aprender ciencias. Revista Educación y Pedagogía, XVIII (45), 61-71.

Martí, J. (2012). Aprender ciencias en la educación primaria. Barcelona: Graó.

Martínez Bonafé, J. (2002). Políticas del libro de texto escolar. Madrid: Morata.

Martínez Losada, C. y García Barros, S. (2003). Las actividades de primaria y ESO incluidas en libros escolares: ¿qué objetivo persiguen?, ¿qué procedimientos enseñan? Enseñanza de las Ciencias, 21(2), 243-264

MECD. (2013). Ley Orgánica 8/2013, de 9 de diciembre, para la mejora de la calidad educativa, BOE núm. 295, 10 de diciembre de 2013.

MECD. (2014). Real Decreto 126/2014, de 28 de febrero, por el que se establece el currículo básico de la Educación Primaria, BOE núm. 52, 1 de marzo de 2014

Megid Neto, J. y Fracalanza, H. (2003). O livro didático de ciências: problemas e soluções. Ciência \& Educação (Bauru), 9(2), 147157.

Miralles P., Gómez Carrasco, CJ. y Sánchez Ibáñez, R. (2014). Dime qué preguntas y te diré qué evalúas y enseñas. Análisis de los exámenes de ciencias sociales en tercer ciclo de Educación Primaria. Aula Abierta, 42(2), 83-89.

Monereo Font, C. (2010). ¡Saquen el libro de texto! Resistencia, obstáculos y alternativas en la formación de los docentes para el cambio educativo. Revista de Educación, 352, 583-597.

Occeli, M. y Valeiras, N. (2013). Los libros de texto de ciencias como objeto de investigación: una revisión bibliográfica. Enseñanza de las Ciencias, 31(2), 133-152.

Patiño Garzón, L., Vera Márquez, ÁV. y Meisel Donoso, JD. (2010). Análisis de la práctica docente desde una experiencia de la Enseñanza de la Ciencia Basada en la Indagación (ECBI). Educere, 14(49), 333-344.
Pérez Martín, JM. y Bravo Torija, B. (2018). Experiencias para una alfabetización científica que promueva la justicia ambiental en distintos niveles educativos. Revista Internacional de Educación para la Justicia Social, 7(1), 119-140.

Pro, A. (2013). Enseñar procedimientos: por qué y para qué. Alambique. Didáctica de las Ciencias Experimentales, 73, 6976.

Puelles, M. (2000). Los manuales escolares: un nuevo campo de conocimiento. Historia de la Educación. Revista Interuniversitaria, 19, 5-12.

Quilez, J. (2006). Análisis de problemas de selectividad de equilibrio químico: errores y dificultades correspondientes a libros de texto, alumnos y profesores. Enseñanza de las Ciencias, 24(2), 219-240.

Quiroz, R. (2001). La educación secundaria en México al inicio del siglo XXI. Educación 2001, 6(70), 21-31.

Robles, A., Solbes, J., Cantó, JR. y Lozano OR. (2015). Actitudes de los estudiantes hacia la ciencia escolar en el primer ciclo de la Enseñanza Secundaria Obligatoria. Revista Electrónica de Enseñanza de las Ciencias, 14(3), 361-376.

Rocard, M., Csermely, P., Jorde, D., Lenzen, D., WalbergHenriksson, H. y Hemmo, V. (2007). Science education Now: A renewed Pedagogy for the future of Europe. Recuperado de https://bit.ly/2PimDOc.

Rodríguez Moreno, J., Pro Bueno, A. y Molina Jaén, MD. (2018). Opinión de los docentes sobre el tratamiento de las competencias en los libros de texto de Ciencias de la Naturaleza en Educación Primaria. Revista Eureka sobre Enseñanza y Divulgación de las Ciencias, 15(3), 3102-3121.

Solbes, J. (2011). ¿Por qué disminuye el alumnado de ciencias? Alambique. Didáctica de las Ciencias Experimentales, 67, 5361.

Solbes, J., Montserrat R. y Furió C. (2007). El desinterés del alumnado hacia el aprendizaje de la ciencia: implicaciones en su enseñanza. Didáctica de las Ciencias Experimentales y Sociales, 21, 91-117.

Torres, J. (1994). Globalización e interdisciplinariedad: el curriculum integrado. Madrid: Morata.

Torres Santomé, J. (1989). Libros de texto y control del currículum. Cuadernos de Pedagogía, 168, 50-55.

Urones, C., Escobar, B. y Vacas, JM. (2013). Las plantas en los libros de Conocimiento del Medio de $2^{\circ}$ ciclo de primaria. Revista Eureka sobre Enseñanza y Divulgación de las Ciencias, 10(3), 328-352.

Verde Romera, AM., Caballero Caballero, I. y Pablos Miguel, M. (2017). La competencia cientifica en los textos escolares. Un estudio LOE-LOMCE. Enseñanza de las Ciencias, Número extra, 1129-1134. 